\title{
VOLODYMYR MYKHAILOVYCH MAZUR (to 75th anniversary of his birthday)
}

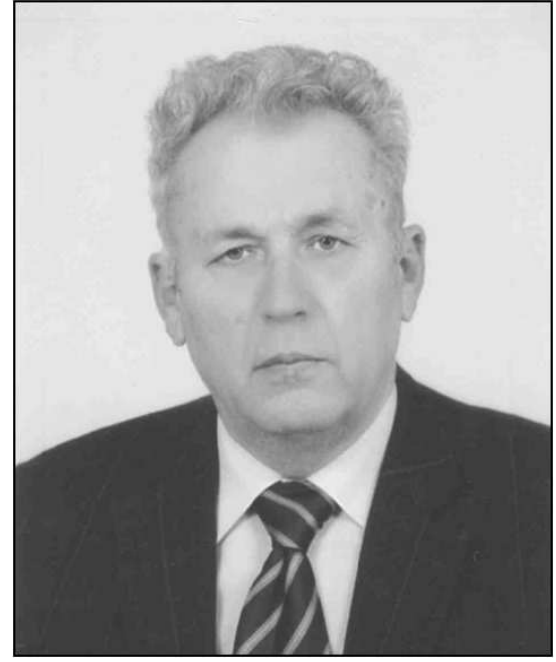

On July 7, 2018, Volodymyr Mykhailovych Mazur the well-known scientist, an outstanding specialist in nuclear physics, Laureate of the State Prize of Ukraine in science and engineering, Dr.Sci. in physics and mathematics, the Chief scientific researcher of the Institute of Electron Physics of the National Academy of Sciences of Ukraine - celebrates his 75th birthday.

Volodymyr Mykhailovych was born in Mukachevo, the Zakarpats'ka region. In 1961, he finished a secondary school with a gold medal and, the same year, entered the Faculty of Physics and Mathematics of the Uzhgorod State University (UzhSU). In 1962, he was called to the Soviet Army, and after the demobilization in 1965, he continued his studies at the Faculty of Physics of the UzhSU, at the Chair of Nuclear Physics. It should be noted that the student training level at this Chair was very high. In particular, the course of nuclear physics lectured at the chair was analogous to that lectured at the Moscow State Uni-

(C) G.M. GOMONAI, O.B. SHPENYK, V.T. MASLYUK, A.M. ZAVILOPULO, 2018

ISSN 2071-0194. Ukr. J. Phys. 2018. Vol. 63, No. 6 versity. The students had probation at leading scientific centers of the USSR and abroad. In 1967, the future scientist had an induction practice at the Košice and Prague Universities in Czechoslovakia. In 1968, he worked on probation at the Kharkiv Institute of Physics and Engineering on the $1.2-\mathrm{GeV}$ linear electron accelerator, the largest in Europe.

V.M. Mazur performed his graduate work at the Laboratory of Nuclear Reactions of the Joint Institute for Nuclear Research (Dubna, Russia). The Laboratory was headed by one of the leading nuclear scientists of the USSR, Academician G.N. Flerov. In 1969, Volodymyr Mykhailovych graduated with honors from the Uzhhorod University and was soon employed as an engineer at the Chair of Nuclear Physics into a group working on the electron accelerator, a microtron M-10. Here, under the supervision of the Chair head Prof. V.A. Shkoda-Ulyanov, a number of works were executed dealing with the activation analysis and radiation physics.

In 1973, V.M. Mazur entered the target postgraduate course at the Laboratory of Photonuclear Reactions of the Institute for Nuclear Research of the Academy of Sciences of the USSR (LPNR INR AS USSR, Moscow), which was the leading photovoltaic center of the country. L.E. Lazareva, Dr.Sci. in physics and mathematics, the founder and the permanent head of this laboratory, was a supervisor of his Ph.D. thesis. The name of L.E. Lazareva is inseparably linked with the development of the world photonuclear science. She was a founder of the works on photonuclear reactions, which are now classical. L.E. Lazareva proposed a classical method to measure the total photoabsorption cross-sections, which turned out to be very fruitful and has been widely used till now in leading laboratories throughout the world. In the early 1970s, the method of total absorption was shown to be applicable to medium and heavy nuclei, including actinides. Volodymyr Ma- 
zur - the young scientist and postgraduate student took an active part in those works. A high-intellectual atmosphere dominated at the laboratory. Links with the leading world's photonuclear laboratories were maintained. It is also worth noting that, near L.E. Lazareva's office, there was an office of a winner of the Nobel Prize in Physics Academician P.O. Cherenkov, who often entered the Laboratory of photonuclear reactions. Laboratory seminars were attended by one of the leading theorists of the USSR, Academician M.O. Markov. A huge scientific authority of the Laboratory of photonuclear reactions and L.E. Lazareva herself favored the world recognition of the results obtained. The years of the postgraduate study of the young scientist Volodymyr Mazur not only created a sound foundation for the future fruitful scientific work, but also transformed into a long-term fruitful cooperation with colleagues from the LPNR INR AS USSR, which allowed him to obtain a number of important results in the future.

In 1976, V.M. Mazur returned to Uzhgorod and began to work at the position of a senior engineer of the Department of photonuclear processes at the Institute for Nuclear Research of the Academy of Sciences of the UkrSSR. In 1979, he successfully defended his Ph.D. thesis "The cross-section of total nuclear absorption of gamma-quanta by heavy nuclei with $A=154 \div 209$ in the region of the giant E1 resonance" at the INR AS USSR (Moscow). In 1980, he was elected to the position of a senior scientific researcher.

In 1981, the Department of Photonuclear Processes became a part of the Uzhhorod Branch of the Institute for Nuclear Research of the Academy of Sciences of the UkrSSR. On the basis of the latter, in 1992, the first independent academic institution in Zakarpattya, the Institute of Electron Physics of the National Academy of Sciences of Ukraine, was created. At this institute, V.M. Mazur worked as a senior scientific researcher, leading scientific researcher (since 1995), and chief scientific researcher (since 2000). In 1995, V.M. Mazur presented his doctoral dissertation "Giant dipole resonance and excitation of isomeric nuclear states at their decay", which he successfully defended at the Institute for Nuclear Research of the National Academy of Sciences of Ukraine. In 1999, V.M. Mazur together with other collaborators from the Institute for Nuclear Research of the National Academy of Sciences of
Ukraine became a winner of the State Prize of Ukraine in science and engineering for a series of works "Regularities and abnormal phenomena in nuclear processes".

The wide scope of scientific interests of V.M. Mazur has determined the versatile character of his scientific activity. The specialists are well aware of the basic directions of scientist's work. They belong to modern nuclear physics. This is the study of the processes of inelastic nuclear scattering of gammaquanta, the study of cross-sections of photonuclear reactions with the excitation of isomeric states in daughter nuclei, the study of the cross-sections of photonuclear reactions at neutron-deficient nuclei (in astrophysics, they are called $p$-nuclei), the measurement of gamma-quantum nuclear absorption crosssections, and the gamma-activation analysis. The members of V.M. Mazur's group developed and implemented precision methods for measuring the yields of photonuclear reactions and determining their absolute cross-sections. Together with the application of isotopically enriched specimens as experimental targets, those methods allowed a number of world-class results to be obtained. In the cooperation with scientists from the INR AS USSR, V.M. Mazur carried out a series of researches concerning the total absorption spectra of gamma quanta by nuclei in the energy interval 8-20 MeV. For the first time, using the absorption method, the cross-sections were measured for a wide number of nuclei ranging from the deformed samarium-154 nucleus to the spherical bismuth-209 one and the actinide series. On a microtron M-30 at the Institute of Electron Physics of the National Academy of Sciences of Ukraine, the excitation cross-sections of isomeric states at the inelastic scattering of gamma quanta within the energy interval 4-15 MeV were measured for the first time for about fifteen isotopes from selenium-77 to mercury-199. In the works by V.M. Mazur, a systematic approach was realized to the study of isomeric ratios in the reactions $(\gamma, n)^{m}$, which made it possible to establish, also for the first time, their effective threshold for photoneutron reactions and obtain the experimental isomeric yield ratios and the excitation cross-sections of the isomeric states of fissionproduct nuclei in the energy interval 8-20 MeV for about thirty isotopes from scandium-44 to platinum197. A large body of the obtained experimental material allowed methods for its systematization to be 
proposed and dependences of the population probability of isomeric states on the nuclear mass and the gamma-quantum energy to be obtained. The experimental data were analyzed on the basis of modern theoretical concepts in the framework of the statistical model and the semidirect mechanism of photoneutron reactions.

The data obtained for photonuclear reactions were published in the leading scientific journals of the world. They enriched the nuclear data database of the International Atomic Energy Agency (IAEA). They were confirmed at other laboratories throughout the world and were positively assessed by the scientific community. V.M. Mazur is a co-author of more than 230 scientific works.

A lot of energy was given by Volodymyr Mykhailovych to the scientific and managerial work. Under his scientific supervision, three young scientists defended their Ph.D. theses in the speciality "nuclear physics, elementary particles, and high energies". For many years, he also worked at the professor position at the Chair of Nuclear Physics of the Uzhgorod National University. He lectured a general course "Nuclear physics" and a special course "Interaction of electromagnetic radiation with nuclei". Under his supervision, many students defended their BSc and MSc degrees and now successfully work in various sectors of the national economy.

For many years, V.M. Mazur was a member of the Scientific Council of the National Academy of Sciences of Ukraine on the problem "Nuclear Physics" at the Division of Physics and Astronomy of the National Academy of Sciences of Ukraine, a member of the specialized scientific council for the defense of candidate and doctoral dissertations at the Institute for Nuclear Research of the National Academy of Sciences of Ukraine (Kyiv), and a member of the Expert Council of the Higher Attestation Commission of Ukraine.

Volodymyr Mykhailovych Mazur is a standard of a scientist devoted to science. Today, as earlier, he works fruitfully and with inspiration, obtaining new important results. He approaches his 75 th birthday not only with significant scientific achievements, but he is full of new creative plans. We sincerely congratulate dear Volodymyr Mykhailovych with his jubilee and wish him sound health, happiness, creative inspiration, and new achievements in science, which will make the national science more "weighty"!

Received 10.05.18. Translated from Ukrainian by O.I. Voitenko 